\title{
Biocompatibility, Inflammatory Response, and Recannalization Characteristics of Nonradioactive Resin Microspheres: Histological Findings
}

\author{
José I. Bilbao · Alba de Martino · Esther de Luis · Lourdes Díaz-Dorronsoro • \\ Alberto Alonso-Burgos · Antonio Martínez de la Cuesta • \\ Bruno Sangro · José A. García de Jalón
}

Received: 10 February 2009/Accepted: 7 April 2009/Published online: 16 May 2009

(C) Springer Science+Business Media, LLC and the Cardiovascular and Interventional Radiological Society of Europe (CIRSE) 2009

\begin{abstract}
Intra-arterial radiotherapy with yttrium-90 microspheres (radioembolization) is a therapeutic procedure exclusively applied to the liver that allows the direct delivery of high-dose radiation to liver tumors, by means of endovascular catheters, selectively placed within the tumor vasculature. The aim of the study was to describe the distribution of spheres within the precapillaries, inflammatory response, and recannalization characteristics after embolization with nonradioactive resin microspheres in the kidney and liver. We performed a partial embolization of the liver and kidney vessels in nine white pigs. The left renal and left hepatic arteries were catheterized and filled with nonradioactive resin microspheres. Embolization was defined as the initiation of near-stasis of blood flow, rather than total occlusion of the vessels. The hepatic circulation was not isolated so that the effects of reflux of microspheres into stomach could be observed. Animals were sacrificed at $48 \mathrm{~h}, 4$ weeks, and 8 weeks, and tissue samples from the kidney, liver, lung, and stomach evaluated. Microscopic evaluation revealed clusters of 10-30 microspheres (15-30 $\mu \mathrm{m}$ in diameter) in the small vessels of the
\end{abstract}

J. I. Bilbao $(\bowtie) \cdot$ E. de Luis · L. Díaz-Dorronsoro •

A. Alonso-Burgos · A. Martínez de la Cuesta

Department of Radiology, Clínica Universitaria de Navarra,

Universidad de Navarra, Avenida de Pio XII, 36,

Pamplona 31008, Spain

e-mail: Jibilbao@unav.es

A. de Martino · J. A. García de Jalón

Department of Histology, School of Veterinary,

Universidad de Zaragoza, Zaragoza, Spain

B. Sangro

Department of Internal Medicine (Liver Unit),

Clínica Universitaria de Navarra, Universidad de Navarra,

Pamplona, Spain kidney (the arciform arteries, vasa recti, and glomerular afferent vessels) and liver. Aggregates were associated with focal ischemia and mild vascular wall damage. Occlusion of the small vessels was associated with a mild perivascular inflammatory reaction. After filling of the left hepatic artery with microspheres, there was some evidence of arteriovenous shunting into the lungs, and one case of cholecystitis and one case of marked gastritis and ulceration at the site of arterial occlusion due to the presence of clusters of microspheres. Beyond $48 \mathrm{~h}$, microspheres were progressively integrated into the vascular wall by phagocytosis and the lumen recannalized. Eight-week evaluation found that the perivascular inflammatory reaction was mild. Liver cell damage, bile duct injury, and portal space fibrosis were not observed. In conclusion, resin microspheres (15-30 $\mu \mathrm{m}$ diameter) trigger virtually no inflammatory response in target tissues (liver and kidney). Clusters rather than individual microspheres were associated with a mild to moderate perivascular inflammatory reaction. There was no evidence of either a prolonged inflammatory reaction or fibrosis in the liver parenchyma following recannalization.

Keywords Embolization - Experimental .

Radioembolization

\section{Introduction}

Intra-arterial radiotherapy with yttrium-90 $\left({ }^{90} \mathrm{Y}\right)$ microspheres (radioembolization) is a therapeutic procedure exclusively applied to the liver that allows the direct delivery of high-dose radiation to liver tumors by means of endovascular catheters, selectively placed within the tumor vasculature [1]. Like other regional treatments for liver 
tumors, this procedure utilizes the well-characterized dual vasculature of the liver to selectively target tumors larger than $5 \mathrm{~mm}$ that are almost exclusively supplied by blood from the hepatic arterial branches. The small microspheres ( $\sim 30 \mu \mathrm{m}$ in diameter), loaded with ${ }^{90} \mathrm{Y}$, preferentially lodge within the tumor microvasculature, delivering highenergy $\beta$-radiation over a limited range (mean penetration of radiation into tissues, $2.4 \mathrm{~mm}$ ), thereby confining the tumoricidal dose ( $>70 \mathrm{~Gy}$ ) to the immediate proximity of the tumor and sparing the normal liver parenchyma [2].

By contrast, conventional embolization of liver tumors, with either TAE (transarterial embolization) or TACE (transarterial chemoembolization), is usually performed with particles larger than $100 \mu \mathrm{m}$. The particles fill the vessels that feed the tumor, provoking embolization and subsequent tumoral ischemia with or without the delivery of drugs to the tumor environment. Smaller particles for TAE/TACE should be used with much care because of the increased risk of adverse distal embolization due to arteriovenous shunting and particles passing through tumoral and nontumoral capillary bed [3].

Two nonbiodegradable microspheres are now commercially available for radioembolization: glass $20-$ to $30-\mu \mathrm{m}-$ diameter microspheres with an activity of $2500 \mathrm{~Bq}$ per particle (size and activity as provided by manufacturer: TheraSphere; MDS Nordion, Canada) and resin 20- to 60$\mu \mathrm{m}$-diameter microspheres with an activity of $50 \mathrm{~Bq}$ per particle (size and activity as provided by manufacturer: SIR-Spheres; Sirtex Medical, Sydney, Australia). Both devices have been extensively evaluated worldwide as adjuncts to chemotherapy, with encouraging clinical results $[4,5]$. Radioembolization is increasingly being recognized for treating liver tumors with a low rate of complications among experienced teams [6-11]. The majority of adverse events with radioembolization are caused by inadvertent infusion of particles into nondetected extrahepatic vessels.

Nevertheless, a number of questions remain unanswered regarding the observed complications with radioembolization. Radiation-induced liver disease is unlikely to occur [12] even though a more recent paper has described up to $20 \%$ mild to moderate radiation-induced liver disease in cirrhotic patients [13]. Associated portal hypertension [14] as well as gastritis or cholecystitis has also been described, but it remains unclear whether these effects are related to the radiation effects or the embolic effects of microspheres, or to both. Moreover, it is not known whether the interaction between the particles and the vasculature has any adverse effect on healthy liver. For better understanding of these issues, we evaluated the ischemic effects of ${ }^{90} \mathrm{Y}$-free (nonradioactive) resin microspheres using a well-known pig model for embolization [15-18]. Embolization of the kidney is a good model to evaluate the depth of penetration of microspheres in the tissue and the resulting effects of embolization as well as the particles themselves. We also studied embolization of the liver, an organ with dual blood supply, as it is the target organ for radioembolization. However, our injection endpoint differed from clinical practice, as we wanted to reach near-stasis in order to evaluate side effects due to potential reflux of particles and subsequent embolization of undesired organs. For this reason, the histological study included the right liver lobe and the gallbladder as well as the stomach. We also analyzed the lung in order to evaluate the possible presence of particles that may have passed through physiologic arteriovenous shunts which are present in the healthy (nontumor) liver parenchyma.

\section{Materials and Methods}

We performed a partial embolization of the liver and the left kidney vessels in nine white pigs (Suis scrofa; weight, $\sim 25 \mathrm{~kg}$ ). The study design was approved by the ethics and animal care committee of our institution and all procedures were conducted in accordance with the European Community rules for animal care (Directive EC 86/609). Aseptic measures were employed as in conventional angiographic studies. Anesthesia was induced via an intramuscular injection of azapirone $(10 \mathrm{mg} / \mathrm{kg}$ body weight) (Esteve, Barcelona) and ketamine $(10 \mathrm{mg} / \mathrm{kg}$ body weight; Merial, Barcelona) and inhalatory halothane (0.8\%-1\%; Astra Zeneca, Madrid) combined with oxygen. An electrocardiogram was used to monitor heart rate during the procedure. The femoral artery was percutaneously catheterized and an intra-arterial 4-Fr sheath (Cordis; L Roden, Netherlands) placed into the artery. A 4-Fr cobra catheter (Cordis) was employed to catheterize the left renal and the left hepatic arteries, the latter because the right usually divides proximally. The deployment of particles was made through a 2.7 coaxial microcatheter (Progreat; Terumo, Japan).

For each injection, a volume of resin microspheres equivalent to a standard clinical dose of $3 \mathrm{GBq}$ was prepared. The resin microspheres were dispersed in $20 \mathrm{ml}$ of sterile water for injection into the kidney and $30 \mathrm{ml}$ of sterile water for injection in the liver. Using a three-way stopcock, a 2-ml syringe was filled from the larger syringe ( 20 or $30 \mathrm{ml}$ ). Particles were continuously shaken between the two syringes and then slowly injected into the microcatheter with the 2-ml syringe.

Embolization was defined as the initiation of near-stasis of blood flow. This end point was reached when contrast medium was retained in the capillary network during three successive heartbeats. On average, near-stasis was reached 
after injection of the contents of two syringes for the kidney and three syringes for the liver (equivalent to 8 million and 12 million resin microspheres, respectively). In order to allow the possible retrograde passage of microspheres to nontarget regions, when particles were applied to the left hepatic artery, the gastroduodenal, right gastric, and other extrahepatic arteries were not embolized as is usual practice in the clinic. This important difference from usual clinical practice enabled us to observe the effects associated with reflux of particles into the lungs and stomach.

Pigs were sacrificed $48 \mathrm{~h}(n=3), 4$ weeks $(n=3)$, and 8 weeks $(n=3)$ following embolization. From each pig, 3mm-thick tissue slices were obtained. Seven transverse and radial sections were obtained from the kidney, three or four from each liver lobe perpendicular to the hilum (including the gallbladder). At least six representative samples were also obtained from the cardial and pyloric areas of the stomach, and three to five sections from the lung. All tissue samples were fixed with $10 \%$ buffered formaldehyde, paraffin-embedded, and cut at $4 \mu \mathrm{m}$. They were stained with the eosin/hematoxylin method for microscopic evaluation.

From evaluation of samples of kidney tissue, records were made of clustering of microspheres that could lead to proximal occlusion of vessels as well as the penetration depth of individual microspheres. In the liver, intra-arterial deposition of microspheres (the presence of clusters leading to proximal tissue) was noted, as well as any tissue pathology. Particularly, we observed possible damage to the liver parenchyma, interaction between the spheres and the portal tract, inflammation in the portal tracts, and damage of the biliary tract and gallbladder endothelium. In the lung and stomach, any potential damage due to the presence of resin microspheres was recorded.

Vessels in which particles were found were classified into three categories: small, medium, and large. Small vessels were defined as those up to $30 \mu \mathrm{m}$ (with particles within them), corresponding in the kidney to afferent $(<25$ $\mu \mathrm{m})$ and arcuate (25- to $60-\mu \mathrm{m})$ arteries. Medium-sized vessels were defined as those measuring from 30 to $90 \mu \mathrm{m}$, corresponding to arcuate and interlobar arteries (60$120 \mu \mathrm{m})$. Large vessels were defined as those larger than $150 \mu \mathrm{m}$, corresponding to interlobar and segmental arteries (200-400 $\mu \mathrm{m})$.

In the liver, small-caliber arteries were those from 10 to $30 \mu \mathrm{m}$, medium-sized arteries those from 30 to $100 \mu \mathrm{m}$, and large arteries those $>100 \mu \mathrm{m}$.

\section{Results}

The following section details the histological findings for each time point. Results are summarized in Table 1.
Forty-Eight Hours Postembolization

\section{Kidney}

Macroscopically the kidney showed spotted to coalescent white areas surrounded by hyperemia, corresponding to small areas of acute infarction associated with embolization. An intense inflammatory reaction composed of neutrophils and lymphocytes was associated with the infarcted areas of the kidney parenchyma. When individual microspheres were found within small vessels (glomerular afferent vessels and arcuate arteries) (Fig. 1A), they did not provoke any significant inflammatory reaction around the vessels.

Liver

No macroscopic changes were observed. In the liver, particles were homogeneously distributed inside small-sized vessels, both in large and small portal spaces and in subcapsular arteries throughout the entire left liver lobe. Aggregates inside large and medium-sized vessels were found and were associated with fibrinoid necrosis of the vessel wall. Single microspheres were extensively diffused in small portal spaces as well as in capsular arteries. There were no signs of ischemia or tissue damage in the parenchyma (Fig. 1B) or biliary tract.

\section{Lung and Stomach}

Following administration of spheres into the left hepatic artery, up to 30 spheres were found in the three lung tissue samples due to arteriovenous shunting. They were distributed throughout the lung vasculature and were individually found in the alveolar capillaries, causing mild dilatation and a slight inflammatory reaction (Fig. 1C).

Resin microspheres were found in the vessels throughout all layers of the stomach (from the mucosa to serosa). Single spheres found in the vasculature of the mucosal layer produced mild perivascular inflammation. When aggregates were found in vessels of the serosa, the muscular layer, and, occasionally, the submucosa, they tended to lead to lumen occlusion. Clusters were associated with arterial wall changes and mild periarteritis, except in one case of marked gastritis and ulceration at the site of arterial occlusion (Fig. 1D).

Four Weeks After Embolization

\section{Kidney}

Macroscopic observation of the kidney showed an irregular surface with small white-gray-colored retracted areas of 


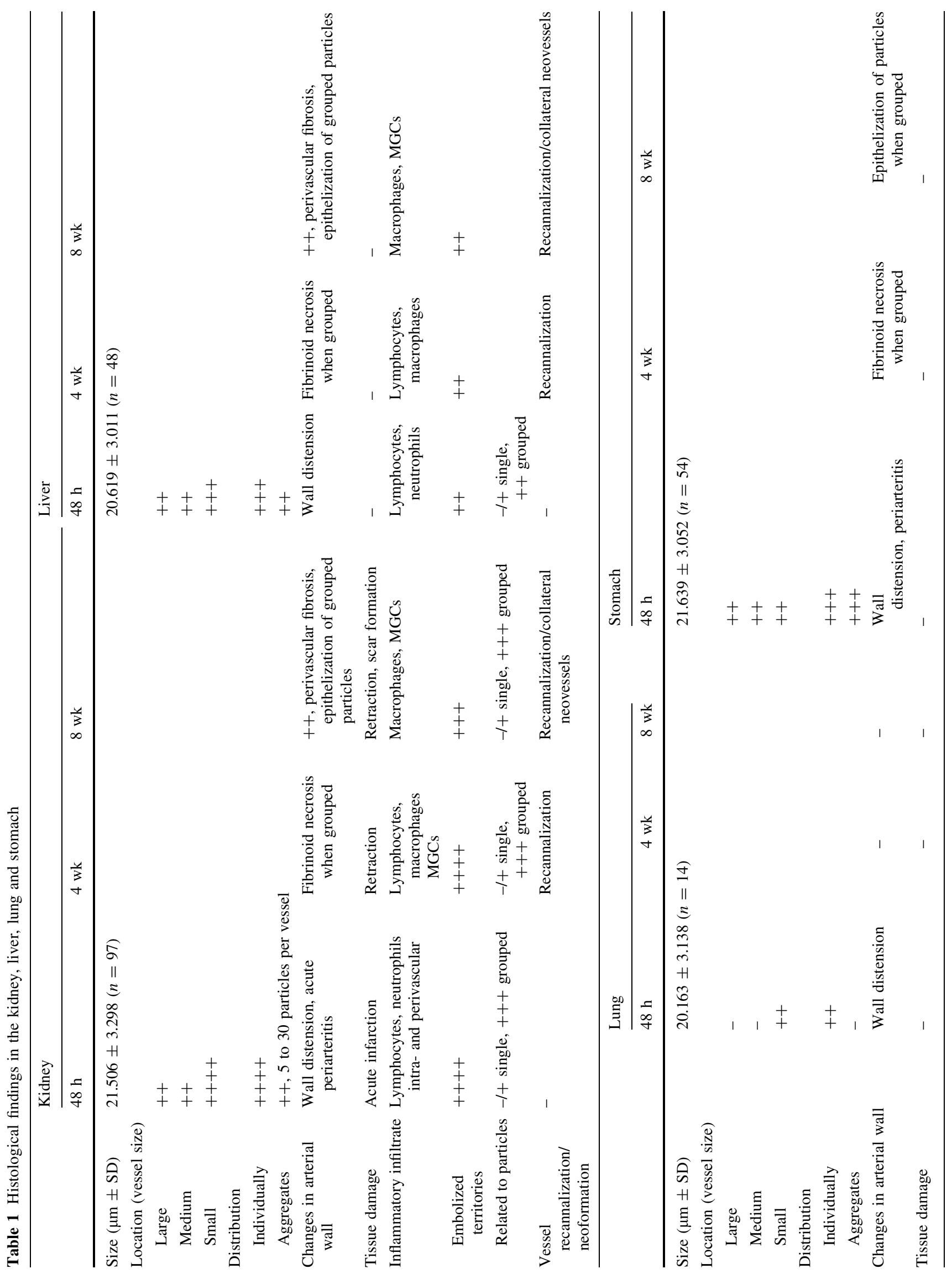


infarction. Microscopically the kidney showed a large number of microspheres (size, $21.4 \pm 3.6 \mu \mathrm{m}$ ) distributed homogeneously within renal parenchyma, mainly in the renal cortex. Two patterns of microsphere distribution were observed: individual spheres inside small vessels and clusters of particles (5 to 30 in number) within mediumsized to large arteries. The most frequent pattern of distribution was individual spheres inside the arcuate arteries, afferent glomerular arteries, and glomeruli. In these cases, perivascular inflammatory reaction was very mild or absent, with macrophage proliferation and a few multinucleated giant cells (MGCs). The microspheres were integrated into the vascular wall and the arterial lumen was recannalized (Fig. 2A).

Liver

Macroscopically, no alterations were observed. Microscopical study showed that microspheres were homogeneously distributed inside small vessels, in both portal tracts and subcapsular arteries of the left liver lobe. Inflammatory reaction was mild or absent and signs of injuries were not found in the surrounding portal space structures or the liver parenchyma (Fig. 2B).

\section{Lung and Stomach}

A small number of spheres were localized in the alveolar walls of the lung.

In the stomach, individual and clusters of microspheres were found in small vessels of the lamina propria next to the epithelium (Fig. 2C), as well as within vessels in the submucosa and muscular layer. Microspheres were found within arteries in the mucosa layer and recannalization of the vessels was observed. The presence of large aggregates of microspheres was associated with a perivascular, inflammatory reaction (mononuclear cells, macrophages, and MGCs).

Two Months After Embolization

Kidney

Macroscopic observation of the kidney revealed an irregular surface with scar tissue retraction and areas of cortical thinning. Microscopic observation showed that microspheres were distributed throughout the kidney parenchyma, but with a higher accumulation in the afferent glomerular arteries and, occasionally, the glomeruli. Compared with observations at $48 \mathrm{~h}$, there were fewer thrombi and microspheres were either embedded in or excluded 
Fig. 1 A Findings at $48 \mathrm{~h}$. Kidney. Two particles within an afferent glomerular artery. No inflammatory reaction associated with this finding was seen. (H\&E; original magnification, $\times 400)$. B Findings at $48 \mathrm{~h}$. Liver. Single sphere in a portal space. No hepatocyte damage or ischemic phenomena were seen. (H\&E; original magnification, $\times 200$ ). C Findings at $48 \mathrm{~h}$. Lung. Particles within an interalveolar septum with very few surrounding inflammatory cells. (H\&E; original magnification, $\times 400)$. D Findings at $48 \mathrm{~h}$.

Stomach. Small particle thrombus (arrow) leading to gastritis and ulceration of gastric epithelium. (H\&E; original magnification, $\times 100$ )
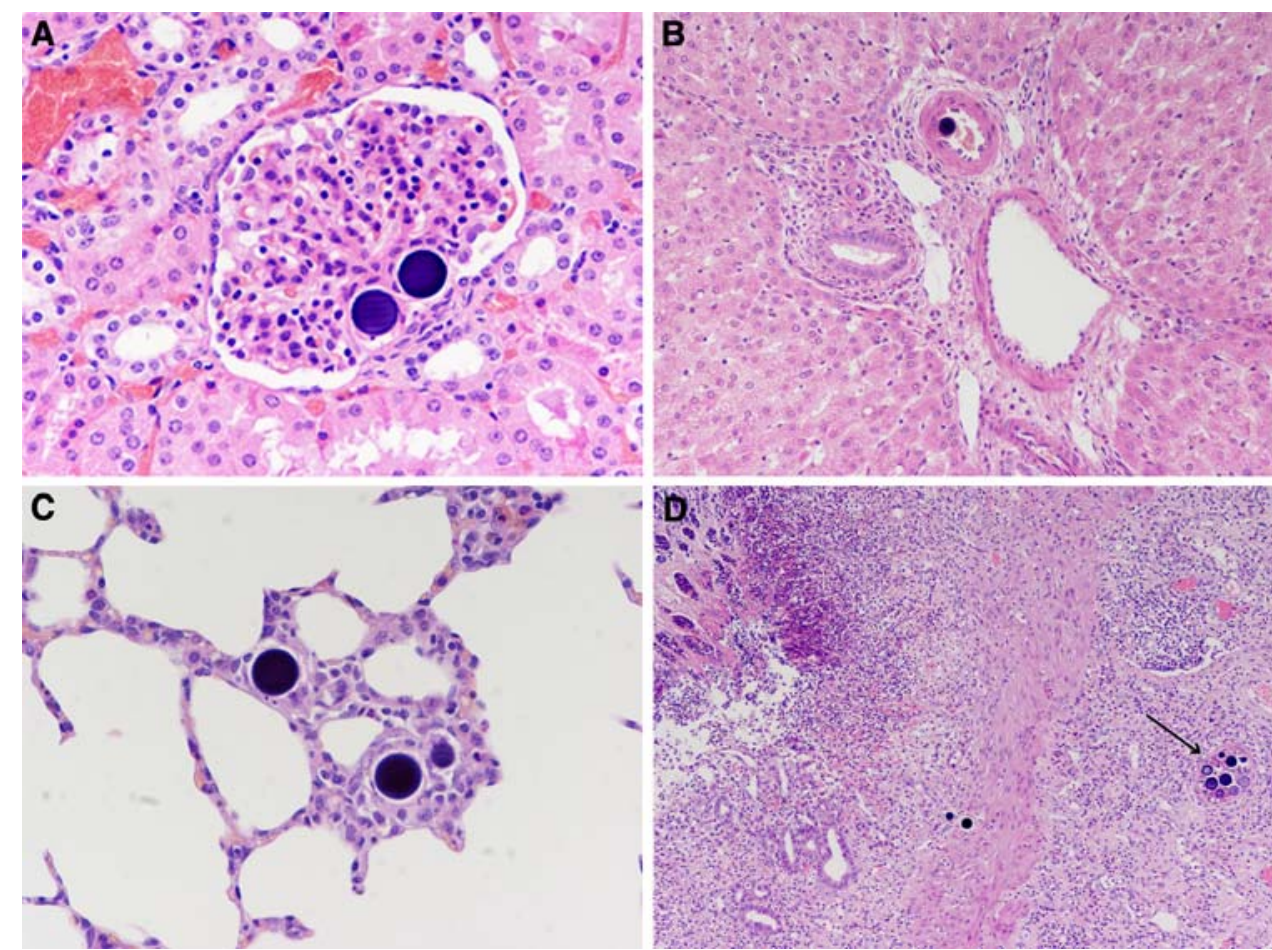

Fig. 2 A Findings at 1 month. Kidney. Particles embedded by the arterial wall. Blood flow was restored by recannalization of vessels (arrow). (H\&E; original magnification, $\times 400)$. B Findings at 1 month. Liver. Particles within a portal space without any associated abnormal histologic finding. (H\&E; original magnification, $\times 200)$. C Findings at 1 month. Stomach. Some spheres are seen inside the lamina propria vessels next to the epithelium. (H\&E; original magnification, $\times 100$ )

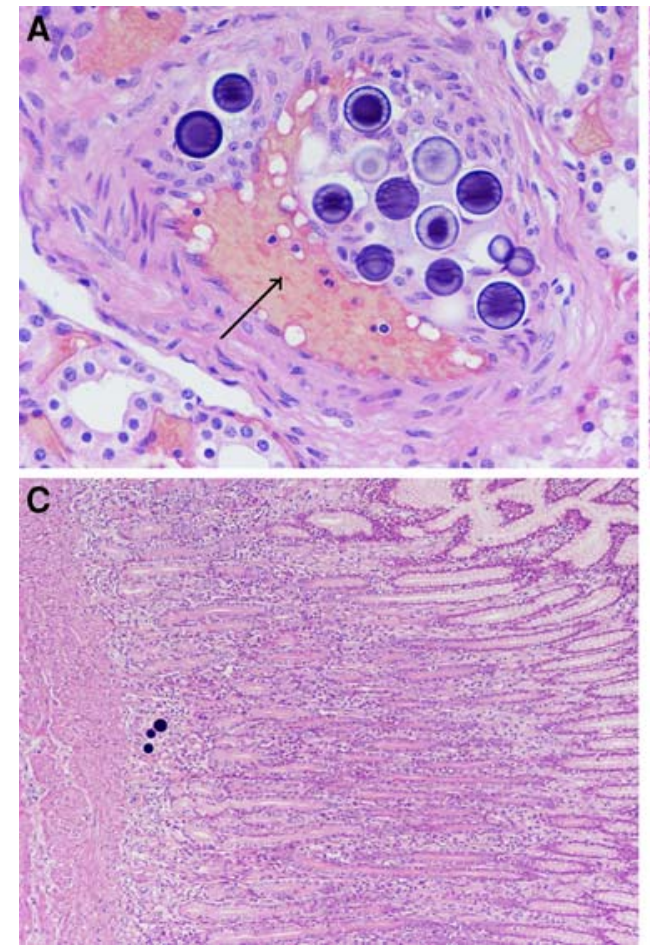

from the arterial walls (Fig. 3A). In the majority of cases, tissues were revascularized following recannalization of the occluded vessel or neovascularization. Associated perivascular inflammatory reaction was mild for both single spheres and clusters consisting of mainly lymphocytic infiltrate and MGCs showing sphere phagocytosis.
Liver

Microspheres were found in vessels of different sizes of the liver reaching the subcapsular arteries. Consistent with observations at $48 \mathrm{~h}$ and 4 weeks, two patterns of distribution were found: single spheres reaching the small portal 
Fig. 3 A Findings at 2 months. Kidney. Complete recannalization of an interlobar artery and exclusion of particles from it (arrow). A slight associated inflammatory reaction was observed. (H\&E; original magnification, $\times 200$ ). B Findings at 2 months. Liver. Recannalization of an artery formerly occluded by a group of particles that have been excluded from the artery (arrow). A very mild inflammatory reaction associated with the particles. (H\&E; original magnification, $\times 400)$. C Findings at 2 months. Liver. Particles within a large portal space. No bile duct damage was noted. (H\&E; original magnification, $\times 200$ ). D Findings at 2 months. Liver. Two particles in a small portal space. Absence of fibrosis and inflammatory reaction. (H\&E; original magnification, $\times 400$ ). E Findings at 2 months. Gallbladder. Inflammation, erosion, and epithelial ulceration of the gallbladder wall due to ischaemia. A particle within the gallbladder lumen is visible. (H\&E; original magnification, $\times 100)$. $\mathbf{F}$ Findings at 2 months. Lung. Particles within an interalveolar septum without surrounding inflammatory cells. (H\&E; original magnification, $\times 400$ )
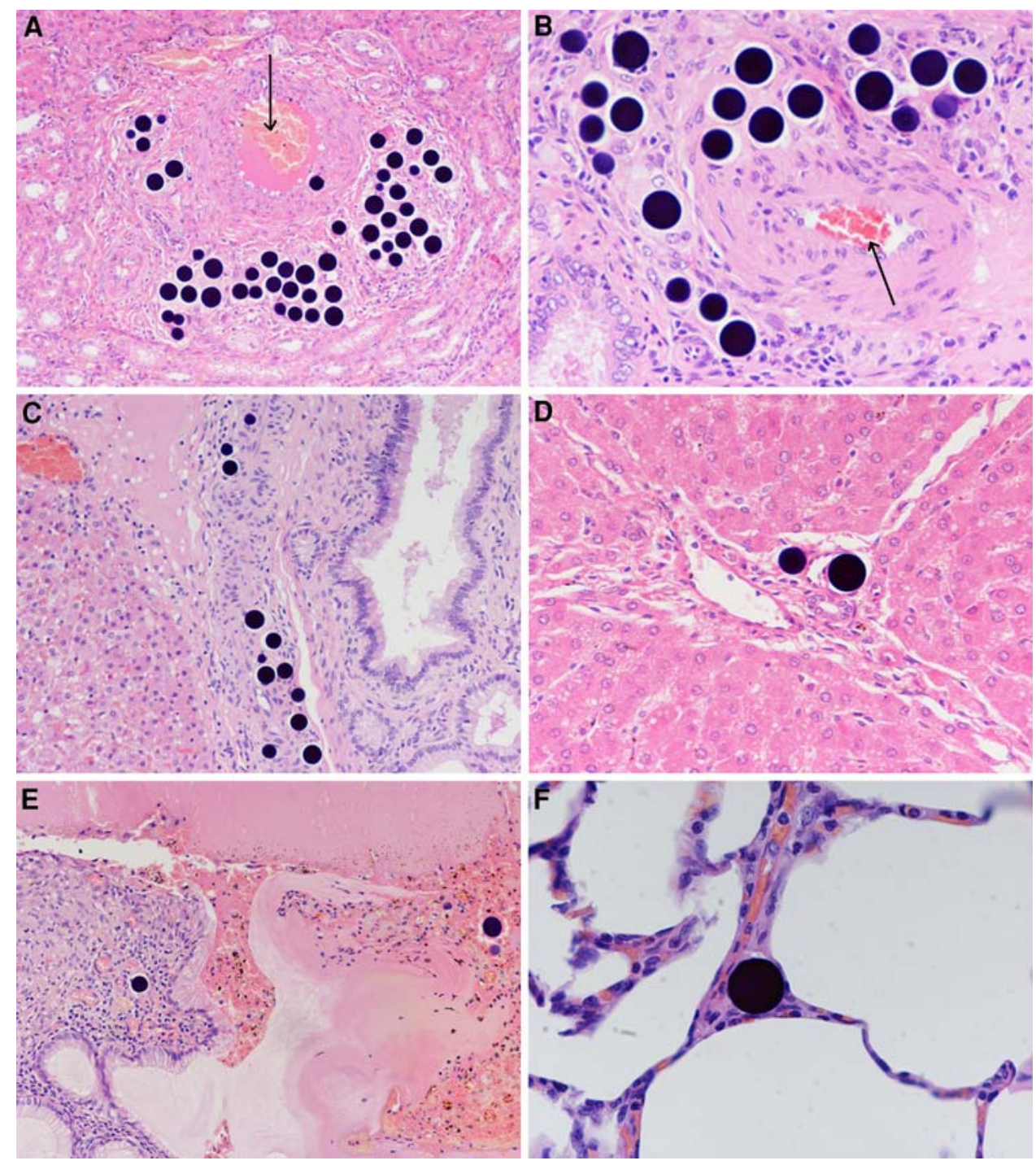

spaces and, less frequently, aggregates of microspheres in the large portal tracts. Microspheres were embedded in the arterial wall, leading to vessel recannalization (Fig. 3B). Perivascular inflammatory reaction was mild and neither liver cell damage, bile duct injury (Fig. 3C), nor portal space fibrosis (some portal tracts showed slight fibrosis) was observed (Fig. 3D). One case of intense cholecystitis occurred and was associated with epithelial ulceration, thrombosis, ischemia, and inflammation. In this last case, a large number of individual spheres were seen within the gallbladder wall, some reaching the gallbladder lumen (Fig. 3E).

\section{Lung and Stomach}

Very few microspheres were located within the lung interalveolar septum arterioles. No inflammatory reaction was observed (Fig. 3F).
In the stomach, individual spheres were located inside the serosa, the muscular layer, the submucosa, and the lamina propia, but without an associated inflammatory reaction. Aggregates were also found within the muscular and submucosa layers of the arteries. Blood flow was re-established and particle aggregates were surrounded by a mild foreign body reaction, with mononuclear and multinucleated cells.

In summary, the following observations were made. The size of the microspheres ranged between 18 and $25 \mu \mathrm{m}$. Microspheres were found in the small arteries and arterioles. Clusters of microspheres tended to be associated focal ischemia of the vascular walls with a mild to moderate perivascular inflammatory reaction. Aggregates were found in all specimens, with the exception of the lung. For the most part, individual microspheres did not provoke either vessel wall damage or a perivascular inflammatory reaction.

After 1 to 2 months, particles tended to be incorporated within the vessel wall, causing a mild extravascular foreign 
body reaction. With the exclusion of microspheres from the vessel lumen, blood flow was re-established due to either vessel recannalization or neovascularization (new vessel formation).

We found one case of cholecystitis as well as one case of marked gastritis and ulceration at the site of arterial occlusion due to the presence of clusters of microspheres.

Ischemic sequelae were mild to moderate in the kidney and almost absent in the liver.

\section{Discussion}

Radioembolization is an effective method for inducing liver tumor regression. The results of several clinical studies have supported its use and confirmed its efficacy in the treatment of primary [10, 11] and metastatic [7-9] liver neoplasm. The incidence of complications after radioembolization is low, if patients are selected appropriately and target delivery is performed meticulously [2]. Gastrointestinal complications have been reported to occur in $3 \%$ to $29 \%$ of patients treated with radioembolization [19], however, little is known about the mechanism of injury underlying these complications [19-21]. Similar gastrointestinal complications have also been observed after chemoembolization [22] and intraarterial infusion of chemotherapy [23]. Gastric and duodenal ulcerations are caused by untargeted delivery of particles in the gastroduodenal territories via extrahepatic visceral arterial branches [2]. In radioembolization, as in other intraarterial liver procedures, this risk can be minimized by occluding extra hepatic visceral arteries prior to administration of microspheres [24, 25].

Although radioembolization is, technically, an embolization procedure since particles are administered intraarterially, and vascular occlusion is expected, the aim of the procedure is not to occlude vessels but to deliver a lethal dose of radioactivity in the tumor via its arterial/ arteriolar network. Maintaining adequate oxygenation of the tumor tissue increases the lethal effect of the radiation, and an important ischemia, due to a large tissue embolization, could then limit the efficacy of the treatment [1]. Additionally, it is important that particles reach the peritumoral and intratumoral vessels, in order to deliver ${ }^{90} \mathrm{Y}$ as close as possible to the tumor cells and to limit proximal occlusion and extratumoral irradiation. For these reasons, particles must be small enough $(<30 \mu \mathrm{m})$ to reach the tumor microvasculature. Clustering of microspheres (scarcely observed in our study) could be due to an unavoidable intraprocedural arterial spasm but also to an inherent tendency of the microspheres to aggregate.

As expected in the kidney (an organ with terminal arterial vascularization), the acute vascular occlusion caused ischemia and infarct of the parenchyma. Clusters, rather than individual microspheres, were found to provoke a mild to moderate perivascular inflammatory reaction. Individual particles found as distally as in the intraglomerular arteries were not found to provoke any kind of local reaction.

In the liver, particles were initially found within the vascular lumen, without any associated damage to the bile ducts or parenchyma. At 2 months, most of the microspheres had been excluded from the vessel lumen, being either incorporated into the vessel wall or completely excluded from the arteries. When particles were excluded into the perivascular tissue, a mild perivascular foreign body reaction was observed, associated with a recannalization of the vessel.

Recent experimental studies [16-18], performed with several embolic particles (size, between 40 and $900 \mu \mathrm{m}$ ), have demonstrated that the presence of a foreign body reaction is generally low, though different between agents. The presence of giant cells was more frequently associated with small sizes, especially after embolization with small Tris-acryl gelatin (Embosphere) particles. The present study demonstrates that, in the liver, the foreign body reaction was very low or even absent with the studied microspheres $(18-25 \mu \mathrm{m})$.

A large number of individual spheres were found in the different layers (muscular, submucosa, and mucosa) of the stomach, with minimal associated inflammatory reaction. When aggregations of particles were found, blood flow was re-established by neovascularization. In one case $(48 \mathrm{~h}$ after the embolization), gastritis associated with arterial occlusion (a cluster of particles) was seen.

All tissue samples showed microspheres within the gallbladder layers. A sample (after 2 months) showed evident histological signs of active ischemic cholecystitis. It is well known that cholecystitis may occur after arterial embolization using particles of different sizes and characteristics. In the radioembolization procedure, microspheres may migrate through the cystic artery to the gallbladder. Although cases of characteristic thick-walled appearance of the gallbladder have been observed and reported, the risk of cholecystitis requiring cholecystectomy is low [26]. In order to avoid this complication, injection should be performed placing the catheter tip distally to the cystic artery. However, numerous anatomical variations of the origin of the cystic artery/ arteries can preclude such positioning of the catheter. The best way to avoid migration of particles into the gallbladder and the resulting complications is still open to debate. This experimental study demonstrates that cholecystitis, caused exclusively by ischemia, may appear after the administration of microspheres.

A few particles were found in the lung in all samples. At 2 days the presence of microspheres was associated with mild dilatation and slight inflammatory reaction of the alveolar capillaries. This inflammatory reaction was not 
observed in subsequent observations at 4 and 8 weeks. In healthy liver parenchyma, particles of small size may pass through direct connections from the hepatic arterioles to the sinusoids, described by Breen et al. as transsinusoidal connections [27]. This could explain why some spheres were found in the lung.

The limitations of this study are, first, that our maximum follow-up was 8 weeks. At this time, spheres seem to be "inert," with no effect on the liver parenchyma cells. However, since particles are excluded from the vessel into the perivascular space and cause an extravascular foreign body reaction, they may, over time, induce fibrosis in the portal spaces. Additional, longer-term evaluation is needed.

The second limitation is the relatively small number of animals (nine), divided into three groups. However, in our view, the information provided about biocompatibility and performance of the microspheres is useful and representative since we obtained samples from the kidney (terminal vessel organ), the liver (with dual blood supply), and all the other viscera that could be involved when a "nontargeted" radoembolization occurs.

Third, it could be argued that our study is limited by the large quantity of particles injected, the endpoint of injection, and the method of injection. However, our objective was to evaluate the embolic effect of these particles. For this reason, we chose near -stasis as the embolization endpoint in order to mimic the worst clinical situation. Our objective was to demonstrate that, even in the worst situation, the damage caused by these particles was limited. However, these results should not be understood as evidence that stasis should be the objective of radioembolization.

In conclusion, when microspheres (18 to $25 \mu \mathrm{m})$ are found individually they occlude distal, small vessels with mild or no associated perivascular inflammatory reaction. Particles are excluded from the vascular lumen by a phagocytosis process, with subsequent recannalization of the embolized arteries. Vascular occlusion has an ischemic effect on organs with terminal vascularization such as the kidney. No ischemic complications were observed in the liver parenchyma in our study. Although infrequent, particles have a tendency to aggregate (10-30 spheres) and occlude proximal vessels; this may lead to ischemic complications (i.e., gastric ulceration). In radioembolization with resin microspheres the complications rate and their severity are mainly related to the irradiating effect rather than the embolizing effect.

\section{References}

1. Kennedy A, Nag S, Salem R et al (2006) Recommendations for radio-embolization of hepatic malignancies using yttrium-90 microsphere brachytherapy: a consensus panel report from the
Radio-embolization Brachytherapy Oncology Consortium (REBOC). Int J Radiat Oncol Biol Phys 68:13-23

2. Campbell AM, Bailey IH, Burton MA (2001) Tumour dosimetry in human liver following hepatic yttrium-90 microsphere therapy. Phys Med Biol 46:487-498

3. Brown KT (2004) Fatal pulmonary complications after arterial embolization with 40-120-microm tris-acryl gelatine microspheres. J Vasc Interv Radiol 15:887-888

4. Sato K, Lewandowski R, Bui J et al (2006) Treatment of unresectable primary and metastatic liver cancer with yttrium-90 microspheres (TheraSphere): assessment of hepatic arterial embolization. Cardiovasc Interv Radiol 29:522-529

5. Pöpperl G, Helmberger T, Münzing W, Schmid R, Jacobs TF, Tatsch K (2005) Selective internal radiation therapy with SIRSpheres in patients with nonresectable liver tumours. Can Biother Radiopharm 20:200-208

6. Gray B, Van Hazel G, Hope M et al (2001) Randomised trial of SIR-Spheres plus chemotherapy vs. chemotherapy alone for treating patients with liver metastases from primary large bowel cancer. Ann Oncol 12:1711-1720

7. Jakobs TF, Hoffmann RT, Dehm K et al (2008) Hepatic yttrium90 radio-embolization of chemotherapy-refractory colorectal cancer liver metastases. J Vasc Interv Radiol 19:1187-1195

8. King J, Quinn R, Glenn DM et al (2008) Radio-embolization with selective internal microspheres for neuroendocrine liver metastases. Cancer 113:921-929

9. Sato KT, Lewandowski RJ, Mulcahy MF et al (2008) Unresectable chemorefractory liver metastases: radio-embolization with $90 \mathrm{Y}$ microspheres. Safety, efficacy and survival. Radiology 247:507-515

10. Sangro B, Bilbao JI, Boan J et al (2006) Radio-embolization using 90-Y resin microspheres for patients with advanced hepatocellular carcinoma. Int J Radiat Oncol Biol Phys 66:792-800

11. Ibrahim SM, Lewandowski RJ, Sato KT et al (2008) Radioembolization for the treatment of unresectable hepatocellular carcinoma: a clinical review. World J Gastroenterol 14:16641669

12. Kennedy A, Coldwell D, Nutting C et al (2006) Resin 90-Y microsphere brachytherapy for unresectable colorectal liver metastases modern USA experience. Int J Radiat Oncol Biol Phys 65:412-425

13. Sangro B, Gil-Alzugaray B, Rodríguez J et al (2008) Liver disease induced by radio-embolization of liver tumours: description and possible risk factors. Cancer 112:1538-1546

14. Jakobs T, Saleem S, Atassi B et al (2008) Fibrosis, portal hypertension and hepatic volume changes induced by intra-arterial radiotherapy with 90yttrium microspheres. Dig Dis Sci 53:2556-2563

15. De Luis E, Bilbao JI, García de Jalón JA, Martínez-Cuesta A, de Martino A, Lozano MD (2008) In vivo evaluation of a new embolic spherical particle (HepaSphere) in a kidney animal model. Cardiovasc Interv Radiol 31:367-376

16. Bilbao JI, De Luis E, Garcia de Jalon JA et al (2008) Comparative study of four different spherical embolic particles in an animal model: a morphologic and histologic evaluation. J Vasc Interv Radiol 19:1625-1638

17. Stampfl U, Stampfl S, Bellemann N et al (2009) Experimental liver embolization with four different spherical embolic materials: impact on inflammatory tissue and foreign body reaction. Cardiovasc Interv Radiol 32:303-312

18. Stampfl S, Stampfl U, Bellemann N et al (2008) Inflammation and recanalization of four different spherical embolization agents in the porcine kidney model. J Vasc Interv Radiol 19:577-586

19. Neff R, Abdel-Misih R, Khatri J et al (2008) The toxicity of liver directed yttrium-90 microspheres in primary and metastasic liver tumours. Cancer Invest 26:173-177 
20. Carretero C, Muñoz-Navas M, Betés M et al (2007) Gastroduodenal injury after radio-embolization of hepatic tumours. Am $\mathrm{J}$ Gastroenterol 102:1-5

21. Thamboo T, Tan K, Wang S et al (2003) Extra-hepatic embolization of Y-90 microspheres from selective internal radiation therapy (SIRT) of the liver. Pathology 35:351-353

22. Hirakawa M, Iida M, Aoyagi K, Matsui T, Akagi K, Fujishima M (1988) Gastroduodenal lesions after transcatheter arterial chemoembolization in patients with hepatocellular carcinoma. Am J Gastroenterol 83:837-840

23. Ravizza D, Fazio N, Fiori G et al (2003) Iatrogenic gastroduodenal ulcers during hepatic intra-arterial chemotherapy. Hepatogastroenterology 50:49-53
24. Lewandowski RJ, Sato KT, Atassi B et al (2007) Radio-embolization with ${ }^{90} \mathrm{Y}$ microspheres: angiographic and technical considerations. Cardiovasc Interv Radiol 30:571-592

25. Cosin O, Bilbao JI, Alvarez S et al (2007) Right gastric artery embolization prior to treatment with yttrium-90 microspheres. Cardiovasc Interv Radiol 30:98-103

26. Lewandoski RJ, Salem R (2004) Incidence of radiation induced cholecystitis in patients receiving Y-90 treatment for unresectable liver malignancies. J Vasc Interv Radiol 15(2; pt 2):S162

27. Breen DJ, Rutherford EE, Stedman B, Lee-Elliot C, Hacking CN (2004) Intrahepatic arterioportal shunting and anomalous venous drainage: understanding the CT features in the liver. Eur Radiol 14:2249-2260 